\title{
Comparación a través del picking en tienda de dos alternativas de entrega en un entorno de servicio a domicilio en supermercados. Área temática: logística en ciudad*
}

doi:10.11144/Javeriana.cc17-44.ctpt

\section{Ricardo Otero-Caicedo}

Pontificia Universidad Javeriana, sede Bogotá.

Correo electrónico: r.otero@javeriana.edu.co

\section{Stevenson Bolívar}

Pontificia Universidad Javeriana, sede Bogotá.

Correo electrónico: s_bolivar@javeriana.edu.co

\section{Nicolás Rincón-García}

Pontificia Universidad Javeriana, sede Bogotá.

Correo electrónico: nicolas.rincon@javeriana.edu.co 
Resumen En Colombia, el comercio electrónico está aumentando considerablemente según cifras de la Cámara Colombiana de Comercio Electrónico, CCCE. En este mercado, las grandes superficies como Jumbo, La 14, Almacenes Éxito y Carulla, entre otras, participan por medio del servicio de entregas a domicilio (Home delivery). Este servicio se compone de 3 etapas principales, que comienzan con la recepción de la orden, continúan con la recolección en el almacén de los productos que componen la orden (order picking) y finalizan con la entrega al cliente (delivery). La eficiencia en los procesos logísticos es esencial para garantizar la rentabilidad de los supermercados en este segmento. En particular, la etapa de order picking es fundamental, ya que representa cerca de la mitad de los costos de bodega. Enmarcado en el proceso picking en tienda, en este documento se presenta y analiza la comparación de dos alternativas de entrega de productos: i) durante el mismo día, ii) en el día siguiente. En el primer caso, los pedidos se despachan a medida que van llegando, siguiendo el criterio FIFO (first in first out) para la asignación de cada orden a cada operario. En el segundo caso, las órdenes se acumulan y se despachan al día siguiente, lo que permite agrupar las órdenes en lotes (batching) y asignar a cada operario uno o varios lotes para realizar el picking. Estas dos alternativas se compararon utilizando simulación por eventos discretos. Los resultados indicaron que sostener al cliente la promesa de entrega durante el mismo día de colocación del pedido, incrementa los costos operacionales de picking en $450 \%$ en promedio.

Palabras clave Entrega a domicilio; recolección de productos; creación de lotes; simulación por eventos discretos

\section{Código JEL C15}

\section{Comparison by Means of Picking in Store of Two Delivery Methods in a Home Delivery Environment in Supermarkets. Subject Area: Logistics in the City}

Abstract In Colombia, electronic commerce is increasing considerably according to figures from the Colombian Chamber of Electronic Commerce, CCCE. In this market, department stores such as Jumbo, La 14, Almacenes Éxito, and Carulla, among others, participate through the Home Delivery service. This service consists of 3 main stages, starting on the reception of the order, continuing with the collection in the warehouse of the products that make up the order (order picking), and concluding with delivery to the customer (delivery). Efficiency in logistics processes is essential to ensure the profitability of supermarkets in this segment. Specifically, the order picking stage is fundamental, since it represents about half of the warehouse costs. Framed in the picking in store process, this document presents and analyzes the comparison between two alternatives of product delivery: i) on the same day, ii) on the following day. In the first case, the orders are dispatched as they arrive, following the FIFO (first in first out) criterion for the assignment of each order to each operator. In the second case, the orders are accumulated and dispatched the next day, which allows batching (grouping orders in lots) and assigning one or several lots to each operator to perform the picking. These two alternatives were compared using discrete event simulation. Results indicated that keeping the promise to the customer of delivery on the same day the order is placed increases the operational costs of picking by an average of $450 \%$.

Keywords delivery; order picking; lot creation; discrete event simulation 


\section{Comparação através do picking em loja de duas alternativas de entrega em um ambiente de entrega em domicílios em supermercados. Área temática: logística na cidade}

\section{Resumo Na Colômbia, o comercio eletrônico vem} acrescentando consideravelmente segundo cifras da Câmara Colombiana de Comercio Eletrônico, CCCE. Neste mercado, as grandes superfícies como Jumbo, La 14, Almacenes Éxito e Carulla, entre outras, participam através do serviço de entregas em domicílio (Home delivery). Este serviço compõe-se de três etapas principais que começam com a recepção do pedido, continuam com a coleta na loja dos produtos que compõem a ordem (order picking) e acabam com a entrega ao freguês (delivery). A eficiência nos processos logísticos é essencial para garantir a rentabilidade dos supermercados neste segmento. Em particular, a etapa de order picking é fundamental, já que representa cerca da metade dos custos de armazenagem. Enquadrado no processo picking em loja, este documento apresenta e analisa a comparação de duas alternativas de entrega de produtos: i) durante o mesmo dia, ii) no dia seguinte. No primeiro caso, os pedidos são processados quando eles chegar, seguindo o critério FIFO (first in first out) para a assinação de cada ordem a cada operário. No segundo caso, as ordens são acumuladas e despachadas o dia seguinte, o que permite agrupá-las em lotes (batching) e assignar a cada operário um ou vários lotes para realizar o picking. Essas duas alternativas foram comparadas utilizando simulação por eventos discretos. Os resultados indicaram que suster ao cliente a promessa de entrega durante o mesmo dia de colocação do pedido incrementa os custos operacionais de picking em 450\% na média.

Palavras-chave Entrega a domicilio; coleta de produtos; criação de lotes; simulação por eventos discretos

\section{Introducción}

Desde la introducción de internet en la vida cotidiana, los establecimientos comerciales han implementado nuevas estrategias para generar valor en su negocio. Los grandes supermercados como Éxito, Carulla y Alkosto, entre otros, iniciaron creando portales de internet que sirvieran de vitrina comercial para que los clientes potenciales pudieran consultar la existencia de productos y comparar diferentes referencias sin salir de casa.

Por otro lado, la expansión del uso de internet ha facilitado la comunicación entre los establecimientos comerciales y el cliente, lo que ha permitido a estos establecimientos dar un paso adicional y ofrecer los servicios de venta por internet y entrega de los pedidos a domicilio. Además, la posibilidad de mantener un contacto cercano con el cliente no solo permite compartir información, sino que genera un nuevo canal de ventas B2C [business to consumer]. Según el Observatorio de Compras Online de Colombia (Cámara Colombiana de Comercio Electrónico, CCCE, 2016), el 76\% del total de los internautas es comprador de algún producto o servicio. Entre el grupo de compradores de productos tangibles, cerca del $22 \%$ corresponde a compras de utensilios para el hogar: comida en conserva, productos empaquetados, implementos de aseo y belleza.

Esta estrategia de ventas ha generado un nuevo segmento de mercado que, según Jan Fabian Ehmke y Ann Melissa Campbell (2014), ha reportado incrementos cercanos al 15\% en los últimos años en Estados Unidos. En el caso europeo, se pronostica que en 2020 los pedidos 
a domicilio representarán un tercio del total de las compras (Javelin Group, 2011). Se espera que las regiones que han incrementado su generalización del uso de celulares inteligentes, también tengan incrementos en este mercado. A pesar de las grandes expectativas, es necesario reconocer que esta nueva modalidad trae bastantes retos logísticos en la cadena de abastecimiento.

Johan Visser, Toshinori Nemoto y Michael Browne (2014) mencionan que las entregas a domicilio suelen generar problemas logísticos adicionales que deben ser costeados por los vendedores. Por ejemplo, si un cliente no está en el punto de entrega, el pedido debe ser reprogramado; de igual forma, si se presentan entregas incompletas debido a desabastecimiento de último momento, los productos faltantes deben ser reprogramados. Por otra parte, J. H. Ron van Duin, W. de Goffau, Bart Wiegmans, Lori A. Tavasszy y Maurice Saes (2016) mencionan problemas adicionales relacionados con la gran cantidad de vehículos pequeños que se usan para la entrega de pedidos. Durante los últimos segmentos de las rutas, los vehículos deben recorrer grandes distancias con poca carga, lo cual incrementa los costos de combustible y genera un mayor impacto ambiental.

Adicionalmente, según Bruno Durand y Jesús González-Feliu (2012), el comercio electrónico de productos del hogar tiene aún varios obstáculos para penetrar en el mercado: (i) usualmente, los clientes perciben que los productos vendidos por internet tienen un costo adicional para solventar la implementación de los sistemas de información, (ii) se ha reportado que los productos comestibles y del hogar suelen tener una rentabilidad de tan solo el $2 \%$ (Boyer \& Hult, 2006), por lo que son susceptibles a los incrementos en su costo de entrega. Además, en nuestro entorno, podríamos mencionar un problema adicional: (iii) los portales web no suelen adaptarse a la velocidad del inventario en las tiendas, por lo que pueden contener información no actualizada sobre disponibilidad de productos.

A pesar de las posibles complicaciones del servicio a domicilio, esta es una alternativa atractiva que le brinda al cliente los siguientes beneficios: (i) reducir el tiempo de elección de productos, (ii) evitar el desplazamiento al supermercado y el estacionamiento, (iii) reducir el tiempo de búsqueda de los productos, (iv) eliminar el tiempo de espera en caja y facturación. Junto a esto, los servicios ofrecidos por los portales web reciben transacciones las 24 horas del día y permiten tener un historial de los productos previamente comprados.

Por lo anterior, el segmento de compras por internet y entregas a domicilio se ha convertido en una opción de los establecimientos comerciales para incrementar su participación de mercado, mejorar sus ventas y generar fidelización de clientes (Saskia, Mareï \& Blanquart, 2016). Adicionalmente, Shu-hsien Liao, Yin-ju Chen y Yi-tsun Lin (2011) concluyen que este canal de ventas genera una nueva forma de hacer mercadeo. La implementación de las ventas en línea permite capturar información demográfica, historial de compra e información de contacto que se puede utilizar para generar ofertas personalizadas.

La entrega de pedidos a domicilio ha generado necesidades logísticas adicionales para sopor- 
tar los cortos tiempos de entrega al cliente y la incertidumbre en la cantidad y conformación de pedidos. En particular, requiere procesos para la recepción de los pedidos, políticas de consolidación de los pedidos cuando sea posible, estrategias para la recolección de los productos en las estanterías y herramientas para la distribución al lugar seleccionado por el cliente.

Debido a la gran competencia existente en supermercados y grandes superficies, el servicio de entrega a domicilio debe garantizar la optimización de sus costos logísticos de operación. Los vendedores que ofrecen esta alternativa se ven en la necesidad de crear estrategias de picking y distribución que puedan apalancar los tiempos de entrega prometidos al cliente. Esta investigación se enfoca en los procesos operativos necesarios para realizar el picking de los pedidos.

Los supermercados que ofrecen este tipo de servicio tienen dos alternativas de configurar el picking: con centros de acopio y distribución dedicados o directamente de las estanterías de las tiendas. En las principales cadenas de supermercados que ofrecen este servicio en el Reino Unido, los supermercados realizan el picking directamente de las estanterías de exhibición de productos. Esto les permite reducir los costos de inversión en infraestructura mientras logran tener el volumen necesario para sostener una operación dedicada. Bajo esta situación, la estrategia logística debe garantizar políticas adecuadas que permitan agrupar los pedidos del cliente para facilitar su recolección en tienda y generar rutas en las estanterías para la recolección de productos que minimicen la distancia total recorrida.
Tradicionalmente, para la colocación del pedido, el cliente ingresa a la página web del establecimiento, selecciona los productos que desea y envía la orden de pedido. Como lo mencionan Johan Visser, Toshinori Nemoto y Michael Browne (2014), el cliente puede solicitar que le sea entregado en su residencia o en el punto de venta; las formas más frecuentes de procesamiento de pedidos son:

- Mismo día: el picking de los productos se realiza tan pronto llega la orden y se envía inmediatamente. En esta alternativa, para acortar los tiempos de entrega es usual disminuir el número de intermediarios en el proceso de distribución usando una flota propia.

- Entrega en los siguientes días: los pedidos se envían usualmente el día siguiente. Cada pedido se acomoda en lotes para facilitar el proceso de picking y distribución. Bajo esta modalidad pueden intervenir terceros en la entrega final de los pedidos.

Estas promesas en los tiempos de entrega al cliente pueden afectar directamente los costos de operación del proceso de picking. En especial, la política de consolidación de pedidos en lotes, pues bajo la segunda promesa se tiene certeza sobre la totalidad de los pedidos, lo cual permite agruparlos de manera que se reduzcan los tiempos necesarios para la recolección de los productos solicitados. Por lo tanto, es esencial analizar el impacto de la promesa del tiempo de entrega para la operación logística. Además, se requiere identificar los diferentes escenarios bajo los cuales una alternativa es más rentable que otra. Esto permite que los 
supermercados puedan seleccionar sus promesas de tiempos de entrega, según la expectativa de rentabilidad que hayan definido.

Para analizar el impacto de la implementación de las diferentes promesas de entrega al cliente, se propone un modelo de simulación en FlexSim de un supermercado. La simulación es utilizada para evaluar bajo diferentes escenarios el impacto en costos del proceso de picking de cada una de las alternativas.

La necesidad de evaluar y analizar este segmento de mercado emergente obedece a que varias empresas han fracasado por un mal manejo de la operación logística. Algunos ejemplos son Webvan, Streamline o HomeGrocer, que entraron al negocio de entregas a domicilio, pero tiempo adelante generaron pérdidas por más de 5.000 millones de dólares (Boyer \& Hult, 2006). Según la revisión de literatura, este artículo es el primero en explorar desde la simulación, el impacto en las operaciones de picking bajo diferentes promesas de tiempo de entrega de los pedidos al cliente.

La estructura de este artículo continúa con una revisión de literatura sobre las entregas a domicilio y picking. Posteriormente, en la sección de métodos, describe en detalle las políticas de picking aplicadas en cada uno de los escenarios. Finalmente, se presentan las conclusiones obtenidas en la simulación y las recomendaciones.

\section{Revisión de la literatura}

En los últimos años se ha generado amplia literatura relacionada con el picking. En algunos casos, las investigaciones buscan mejorar la efi- ciencia de los procesos y, en otros, mejorar la percepción del cliente sobre el servicio. En esta última categoría, se tiene el realizado por Kenneth K. Boyer y G. Thomas M. Hult (2006), que evaluaron la percepción frente a dos métodos de order picking mediante una encuesta a 2.100 clientes, incluyendo el nivel de experiencia del cliente y cinco factores: calidad del servicio, calidad del producto, frescura del producto, ahorro de tiempo e intenciones de comportamiento. En el mismo campo, Yi-zhou Chen, Shi Fei Shen, Tao Chen y Rui Yang (2014) evaluaron el grado de satisfacción de las expectativas del cliente contra el tipo de diseño del proceso de picking, mediante la aplicación del método Kansei Engineering.

Usualmente, la simulación es la técnica utilizada para evaluar diferentes acercamientos a problemas relacionados con el picking, debido a su desempeño en presencia de comportamientos estocásticos y con relaciones complejas entre los agentes que conforman el modelo. Heung Suk Hwang y Gyu Sung Cho (2006) utilizando simulación, evaluaron el desempeño de diferentes alternativas de diseño de las instalaciones para un centro dedicado en términos del tiempo requerido para el picking con demanda estocástica. Pyung-Hoi Koo (2009), usando simulación, comparó el desempeño de la metodología bucket brigade (BB) y su propuesta de BB modificada para casos de picking en que esta metodología es aplicable. Adicionalmente, Mengfei Yu y René de Koster (2010), con la simulación, comprobaron que el almacenamiento dinámico, donde solo los productos requeridos para las órdenes actuales se almacenan dinámicamente en el área de picking y los demás en un 
“área de reserva”, aumenta considerablemente el rendimiento del picking y disminuye los costos de operación.

En la etapa de recepción de órdenes se han desarrollado nuevas tecnologías para facilitar este proceso. Adicionalmente, se ha trabajado en mecanismos de aceptación de los pedidos; Dominic Bühler, Robert Klein y Michael Neugebauer (2016) presentan un ejemplo que propone resolver la reserva (booking) de órdenes como un problema lineal mixto. Jan Fabian Ehmke y Ann Melissa Campbell (2014) emplearon técnicas de simulación para evaluar diferentes estrategias de aceptación con el objetivo de no incumplir la promesa básica del tiempo máximo de entrega, dado que, en algunos casos, las empresas plantean como alternativa posponer pedidos (Koster, Le-Duc \& Roodbergen, 2007).

Para la etapa posterior al picking, la distribución, se han empleado técnicas de Vehicle Routing Model (VRM) que incluyen ventanas de tiempo (Bühler, Klein \& Neugebauer, 2016); también se evalúan diferentes alternativas de vehículos y su relación con el tráfico de la ciudad (Park \& Regan, 2004; RincónGarcía, 2016).

En las siguientes subsecciones se hace una revisión más detallada de los dos aspectos más importantes para la política de recolección: consolidación de lotes y selección de rutas.

\subsection{Políticas de recolección (picking)}

El picking se define como el proceso de traer productos del área de almacenamiento en respuesta a un pedido del cliente. Las órdenes de los clientes corresponden a una secuencia de requerimientos, cada uno compuesto de un producto (Stock-Keeping Unit, SKU) y una cantidad. Según René de Koster, Tho Le-Duc y Kees Jan Roodbergen (2007), el picking es una de las actividades que más recursos de horas hombre consumen. Se estima que corresponde al 55\% del total de los costos de operación en los centros de distribución. El proceso de picking es considerado prioritario para incrementar la efectividad de los sistemas logísticos.

La cadena humana, más conocida como bucked, brigada (Bartholdi \& Eisenstein, 1996), es una de las políticas comúnmente implementadas en los sistemas de picking, debido a su capacidad de autogestionarse. Sin embargo, Dieter Armbruster y Esma S. Gel (2006) concluyen que su eficacia medida en la velocidad de recolección de los pedidos radica en los siguientes supuestos: (i) siempre hay un operario más rápido que otro y (ii) los ahorros de tiempo se generan al eliminar situaciones en que dos operarios necesitan recoger el mismo producto al tiempo. Bajo estas condiciones, resulta evidente que el picking dentro de los supermercados no garantiza ninguno de estos supuestos. Cada uno de los operarios será más hábil con el tiempo al reconocer cómo están distribuidos los productos en las estanterías cerca de su zona habitual de trabajo. Los cambios en la velocidad de recolección pueden verse afectados por la rotación de personal. Adicionalmente, debido al pequeño tamaño de los productos, el tiempo adicional que se genera cuando dos operarios requieren recoger el mismo producto es despreciable.

Alternativas adicionales para la generación de las políticas de picking consisten en tomar 
decisiones sobre cómo agrupar los pedidos en lotes y cómo secuenciar el orden de recolección de los pedidos. Estos enfoques resultan más fáciles de aplicar a entornos reales de picking en supermercados, debido a que no perturban la conformación de los pedidos del cliente y son más robustos por la dispersión geográfica de los productos.

\subsubsection{Consolidación de lotes}

De acuerdo con Jason Chao-Hsien Pan, PoHsun Shih y Ming-Hung Wu (2015), la consolidación de lotes es una decisión fundamental dentro del diseño y control de bodegas de abastecimiento y un factor clave para el éxito de las políticas de picking. Básicamente, el problema de consolidación de pedidos (On-Base Percentage, OBP) consiste en determinar cómo los pedidos realizados por los clientes deben ser consolidados en lotes para ser recolectados en un solo viaje, para reducir la distancia total recorrida. Debido a la complejidad computacional de resolver este problema por métodos exactos, se han propuesto varios procedimientos heurísticos para abordarlo. Soondo Hong, Andrew L. Johnson y Brett A. Peters (2012) combinan programación lineal mixta y una heurística basada en un recorrido simulado para consolidar los pedidos en lotes. Chih-Ming Hsu, Kai-Ying Chen y Mu-Chen Chen (2005) proponen un método heurístico basado en algoritmos genéticos que permite consolidar los pedidos de tal forma que se disminuye el tiempo total recorrido y que se puede implementar en cualquier tipo de configuración de bodega. Finalmente, Sebastian Henn y Gerhard Wäscher (2012) presentan dos heurísticas basadas en los principios de búsqueda tabú que logran reducir el número de lotes y la distancia total recorrida en cortos tiempos computacionales.

\subsubsection{Selección de rutas}

Una vez consolidados los pedidos en lotes, se requiere decidir cuál debe ser la ruta de recolección de los productos que minimice la distancia total recorrida. Esta situación puede modelarse con el problema del agente viajero (Travelling Salesman Problem, TSP). Sin embargo, debido a la forma de las estanterías en los centros de distribución, la distancia entre dos puntos depende de la distribución de las estanterías y no puede obtenerse con una distancia euclidiana. Debido a esto, Randolph W. Hall (1993), Soondo Hong y Youngjoo Kim (2017) y Tho Le-Duc y René de Koster (2007) proponen que la ruta de recolección de pedidos se realice recorriendo en forma de $\mathrm{S}$ las estanterías. Esto garantiza la minimización de la distancia total recorrida y además genera una ruta única de fácil recordación para los operarios.

\section{Estructura logística de la entrega a domicilio de productos del hogar}

El servicio de entrega a domicilios de productos del hogar se compone principalmente de tres (3) etapas. Inicia con la recepción de la orden, luego se recogen del almacén los elementos que las componen (order picking) y finaliza con la entrega al cliente (delivery). En este proceso, René de Koster, Tho Le-Duc y Kees Jan Roodbergen (2007) han resaltado como principales 
aspectos a controlar y fuentes de oportunidades de mejora la ubicación de los productos en la bodega o almacén, los métodos de asignación para el almacenaje, los métodos de ruteo interno, la consolidación de pedidos (order batching) y la zonificación. Todos estos aspectos hacen compleja la operación de los supermercados en el segmento de entregas a domicilio, ya que en sus estanterías pueden encontrarse cerca de 30.000 productos diferentes y además, la cantidad de productos pedida por los clientes tiene una naturaleza estocástica.

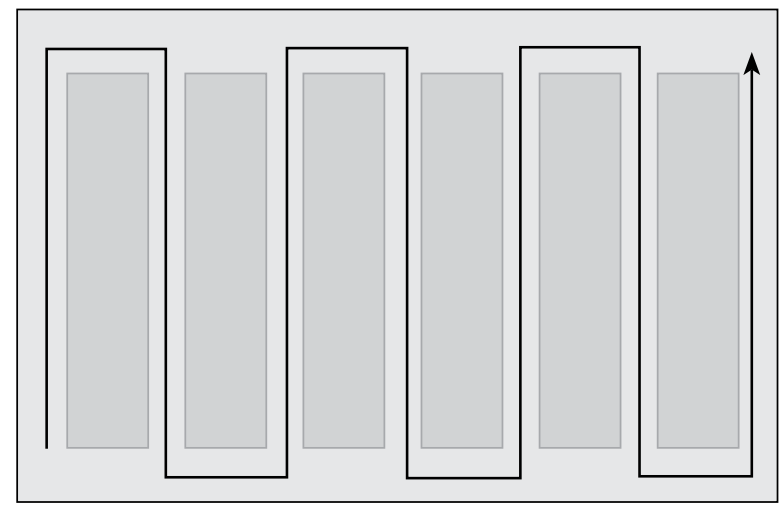

\section{Gráfico 1}

Recorrido del centro de distribución en forma de 'S'

Fuente: elaboración propia

La etapa de recepción del pedido ha evolucionado considerablemente mediante la implementación de sistemas de información web cada vez más ágiles y amigables. Avances como el pago electrónico por internet han impulsado el crecimiento de las compras por este medio. En esta etapa, los principales costos están asociados al mantenimiento del sistema de información y al cobro de entidades bancarias por la captación de los pagos realizados con tarjetas de crédito.

En la etapa de picking se concentra, inicialmente, la distribución de los productos en bodega. En los centros de distribución tradicionales, los productos suelen ubicarse según la magnitud de la rotación de su inventario. Sin embargo, en los supermercados, la distribución de los productos en estantería responde a una estrategia de exhibición a los visitantes, por lo que no se modifica para facilitar la recolección de productos para la entrega de pedidos a domicilio. En esta etapa, también se concentran las políticas de consolidación de pedidos y recolección de productos cuyas actividades impactan directamente en los costos de operación al requerir sistemas de información y horas hombre dedicadas.

Los costos asociados al envío están enfocados al problema de ruteo de vehículos (Vehicle Route Problem, VRP), en el cual influyen el tamaño del camión, el número de camiones y las rutas para realizar las entregas en las ventanas 
de tiempo establecidas. En esta situación, la entrega en la última milla incrementa considerablemente los costos al requerir un elevado número de vehículos que puedan entregar de forma paralela los pedidos a los clientes. Una revisión detallada del VRP y su impacto en las entregas a domicilio se puede consultar en Dominic Bühler, Robert Klein y Michael Neugebauer (2016) y Nicolás Rincón-García (2016).

\section{Definición del problema}

La necesidad de crear procesos logísticos eficientes en el entorno de las entregas a domicilio exige analizar cada una de las etapas que conforman el proceso. Por lo tanto, hay que medir el impacto en los costos operativos de las diferentes promesas de tiempos de entrega al cliente: durante el mismo día y en el día siguiente. Sin embargo, para poder hacerlo, es indispensable definir políticas de consolidación y recolección de los productos que permitan comparar ambas alternativas desde un punto de vista práctico.

\section{Métodos}

Enmarcado en el proceso picking en tienda, se comparan dos alternativas de entrega de productos: i) durante el mismo día, ii) el día siguiente. En el primer caso, los pedidos se despachan a medida que van llegando, siguiendo criterio FIFO (First In, First Out) para la asignación de cada orden a cada operario. En el segundo caso, las órdenes se acumulan y se despachan al día siguiente, lo que permite agrupar las órdenes en lotes (batching) y asig- nar a cada operario uno o varios lotes para realizar el picking.

La política de consolidación de pedidos (batching) se define por medio de una heurística propuesta que busca crear la menor cantidad de lotes posible y minimizar la distancia recorrida en la recolección de los productos de cada uno. En la sección 5.1 se presenta el detalle técnico de la heurística. La secuenciación de los productos a recoger en cada uno de los lotes se define por medio de la ruta de recolección en forma de 'S'. Finalmente, la selección del camino más corto para dirigirse de una estantería a otra se obtiene mediante la heurística Dijkstra. Esta combinación permite representar una forma inteligente de consolidar y recolectar pedidos.

Las dos alternativas se compararon utilizando simulación de eventos discretos, con base en el modelo de un supermercado que cuenta con 12 hileras de estanterías (13 pasillos) cada una de 22,63 metros de largo (11 estanterías), 2 metros de alto y 70 centímetros de profundidad por cada lado. La cantidad de órdenes que se crean en un día se generó mediante una distribución de probabilidad Poisson. Finalmente, la cantidad de productos que conforman una orden se obtuvo por medio de una distribución uniforme discreta.

En los próximos apartados, se presentan en mayor detalle, los métodos implementados durante esta investigación.

\subsection{Heurística de picking}

\subsubsection{Consolidación de pedidos}

Dado un conjunto de órdenes $O$, el problema puede representarse como un Bin packing. 
Se requiere agrupar cada una de las órdenes recibidas durante el día anterior en la mínima cantidad de grupos posible. En esta situación, la capacidad de cada grupo $k$ depende del volumen total que pueda cargar un carrito de compras. Se propone una heurística basada en el algoritmo First Fit (Martello \& Toth, 1987). El total de las órdenes se organiza de forma decreciente según el volumen total ocupado por los productos del pedido. Posteriormente, la primera orden es asignada a un lote. Para disminuir el total de la distancia recorrida por lote, es necesario agrupar pedidos que contengan productos cercanos a los ya asignados. Por lo tanto, se requiere una medida única para representar la posición del lote. Se propone utilizar el centro de gravedad, el cual se define como el promedio de las coordenadas de cada uno de los productos que constituyen hasta el momento el lote.

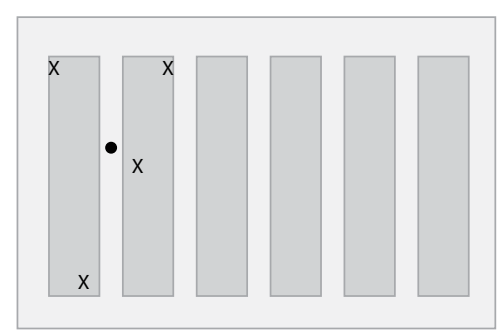

a)

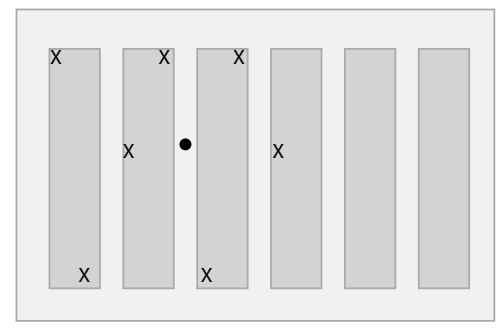

c)

$$
\begin{aligned}
& C G_{X T}=\frac{1}{c(T)} \sum_{\forall i \in T} \operatorname{Pos}_{x}(i) \\
& C G_{Y T}=\frac{1}{c(T)} \sum_{\forall i \in T} \operatorname{Pos}_{y}(i)
\end{aligned}
$$

La ecuación 1 describe el procedimiento de cálculo de la coordenada en el eje horizontal del centro de gravedad del lote $T$ con la candidad de productos $c(T)$ que tiene hasta el momento. La ecuación 2 utiliza los mismos conceptos para obtener el centro de gravedad de la coordenada en el eje vertical. A continuación, se presentan a manera de ejemplo, diferentes centros de gravedad para diferentes órdenes de pedido. En cada gráfica, las X representan la ubicación de un producto y los círculos la ubicación del centro de gravedad.

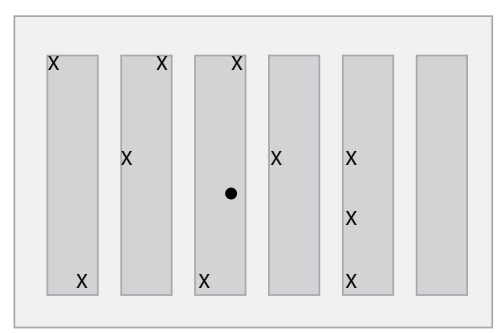

b)

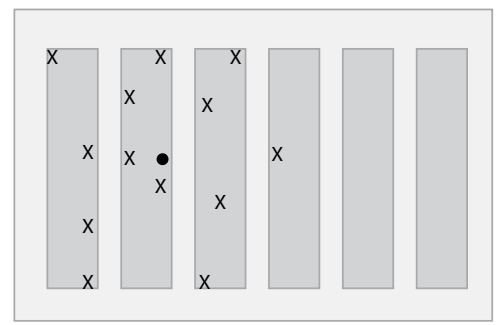

d)

Gráfico 2

Comparación del centro de gravedad de diferentes órdenes de pedido Fuente: elaboración propia 
Las órdenes de pedido representadas en b y d corresponden a los mismos productos iniciales de a y c respectivamente, con algunos productos adicionales. Se puede apreciar que añadir productos cercanos al centro de gravedad garantiza, de cierta manera, que estén cerca de los productos añadidos al lote hasta el momento.
Por lo tanto, el criterio utilizado para agregar un pedido a un lote existente será aquel que tenga la menor distancia promedio de cada uno de sus elementos al centro de gravedad. Este ciclo se repite hasta que todas las órdenes han sido asignadas a un lote. A continuación, se describe el algoritmo de consolidación de pedidos.

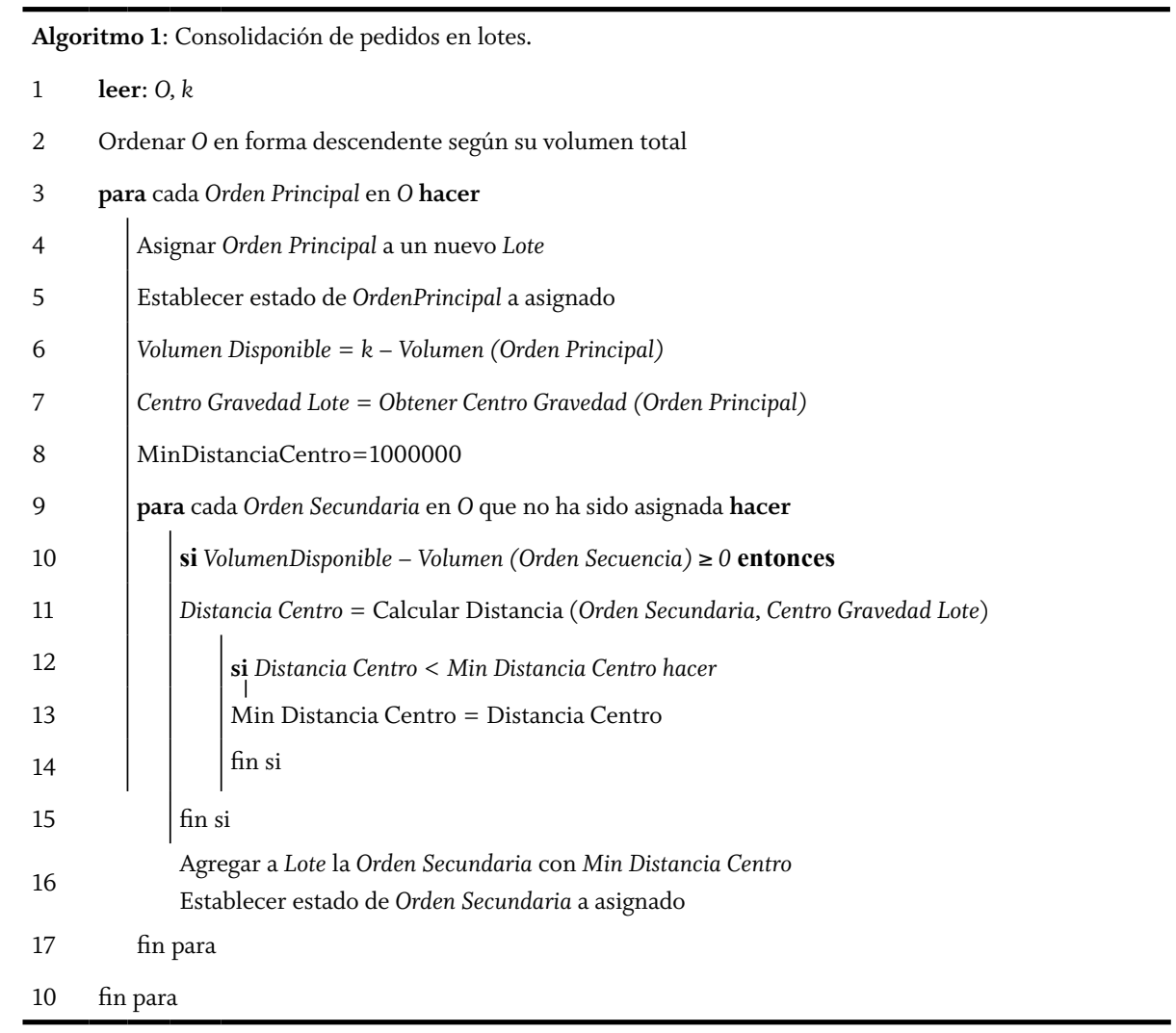

\subsubsection{Creación de rutas para los lotes conformados}

Para la creación de los recorridos, los productos de cada lote son organizados según su posición en la ruta en forma de 'S'. Posteriormente, el operario decide a través del algoritmo Dijkstra (Chen, Shen, Chen \& Yang, 2014) el camino más corto para alcanzar la posición del siguiente producto. La figura 3 muestra el recorrido generado por el método propuesto. Las X representan los productos de un lote que deben ser recolectados y la línea punteada exhibe la ruta de recolección. 


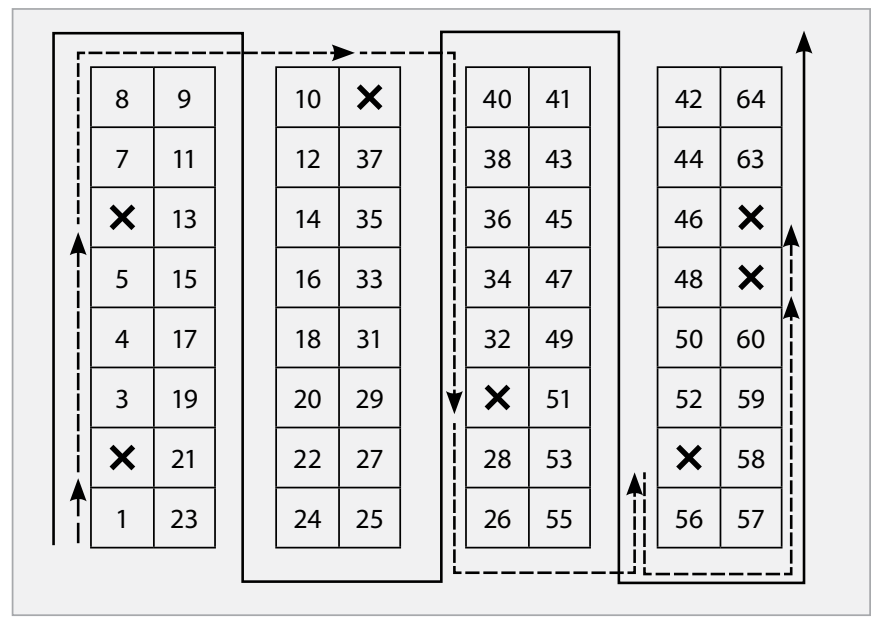

Gráfico 3

Ruta generada según el método propuesto

Fuente: elaboración propia

\subsection{Simulación}

\subsubsection{Escenario de entrega durante el mismo día}

Bajo este escenario, al igual que en René de Koster (1994), se asume que la cantidad de órdenes que llegan cada hora se puede asumir como un proceso de Poisson homogéneo con tasa $\lambda$. Por lo tanto, el tiempo entre llegadas en minutos- de cada orden se puede modelar a través de una distribución exponencial con tiempo promedio $\frac{60}{\lambda}$. Una vez llega la orden, inmediatamente un operario realiza la recolección de los productos solicitados. La generación de rutas se obtiene según la propuesta detallada en la sección 5.1.2.

\subsubsection{Escenario de entrega del día siguiente}

Al inicio de cada día se cuenta con una cantidad de órdenes $n$ que se genera bajo una distribución Poisson con tasa $h \lambda$. Siendo $h$, la cantidad de horas que permanece abierto el establecimiento. Fijar la cantidad de pedidos de esta manera se hace únicamente para hacer comparables las dos alternativas. De esta manera, al inicio de cada día se realiza la consolidación de pedidos en lotes según 5.1.1. Posteriormente se inicia la recolección de cada uno de ellos según las rutas generadas con lo propuesto en la sección 5.1.2.

\subsubsection{Entorno de simulación}

Ambos escenarios se simularon en Flexim ${ }^{\circledR}$, bajo la siguiente distribución común de las estanterías. 




a)

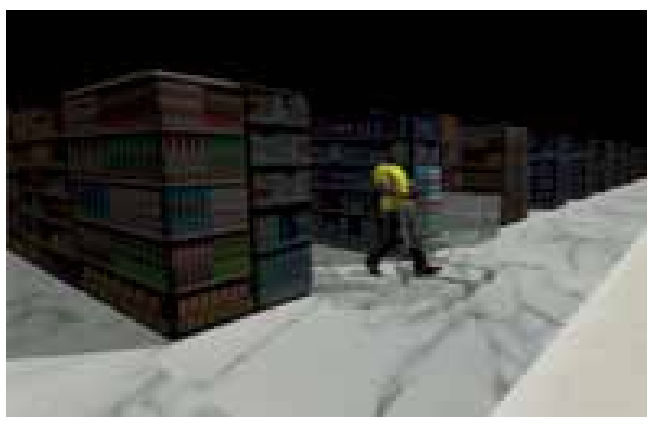

c)

\section{Gráfico 4}

Simulación del proceso de picking en Flexsim ${ }^{\circledR}$

Fuente: elaboración propia

\subsection{Diseño de experimentos}

La comparación de las dos alternativas se llevó a cabo a través de experimentos computacionales. En seguida, se explica cómo se crearon las instancias, las métricas utilizadas para la comparación y la selección de factores y niveles.

\subsubsection{Construcción de las instancias}

La distribución de estanterías está basada en un supermercado local. Existen 230 estanterías que pueden contener 5 tipos de productos diferentes. Es decir, existen 1150 tipos diferentes de productos que un cliente puede seleccionar en un pedido. La cantidad de productos $p_{i}$ se

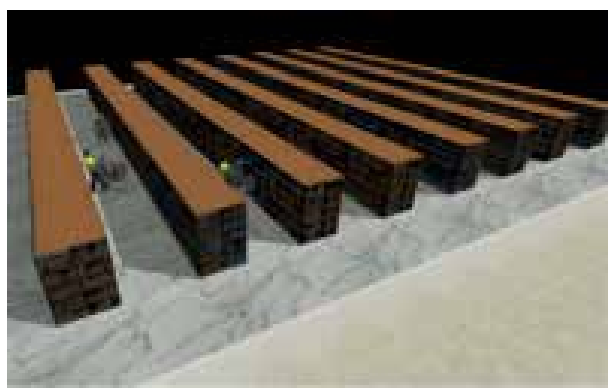

b)

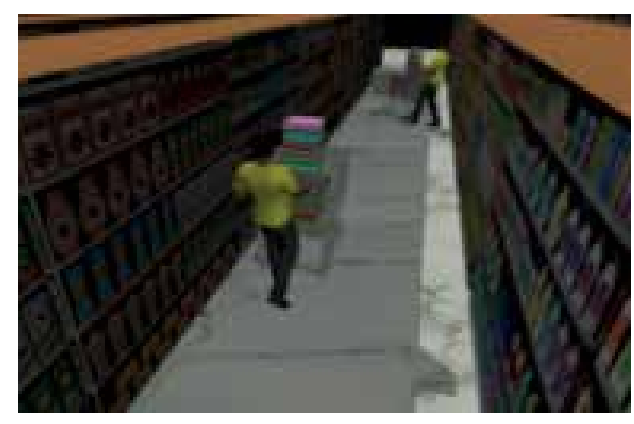

d)

genera bajo una distribución uniforme discreta entre uno y una cantidad fija $M$. Los productos de la orden se seleccionan de forma aleatoria bajo un muestreo sin reemplazo.

\subsubsection{Métricas}

Los costos operativos logísticos asociados al picking en supermercado se miden a través de las horas hombre empleadas para realizar la recolección de los productos de cada lote. Para la comparación de escenarios, se utilizó las horas hombre totales para la recolección de todos los pedidos realizados por el cliente con una cantidad de operarios fija. 


\subsubsection{Factores y niveles}

El propósito de este estudio es comparar las dos alternativas de envío de los productos de los clientes bajo diferentes cantidades de órdenes de pedido. En la tabla 1 se presenta la selección de factores y niveles del diseño de experimentos.

\begin{tabular}{|c|c|c|c|}
\hline Factores a modificar & Niveles & $\begin{array}{c}\text { Factores que no se } \\
\text { modifican }\end{array}$ & Valor \\
\hline Alternativa de envío (A) & $\begin{array}{l}\text { Durante el mismo día (1) } \\
\text { Durante el día siguiente (2) }\end{array}$ & $\begin{array}{l}\text { Número de horas de trabajo al día } \\
\text { (h) }\end{array}$ & 8 \\
\hline $\begin{array}{l}\text { Cantidad promedio de órdenes que se } \\
\text { generan por día }(\lambda / h)\end{array}$ & $\begin{array}{l}100 \\
150 \\
200\end{array}$ & Cantidad de operarios $(R)$ & 3 \\
\hline $\begin{array}{l}\text { Cantidad máxima de productos que un } \\
\text { cliente solicita }(M)\end{array}$ & $\begin{array}{l}10 \\
20 \\
30\end{array}$ & $\begin{array}{l}\text { Capacidad del carrito de compras } \\
\text { (k) }\end{array}$ & $0.15 \mathrm{~m}^{3}$ \\
\hline
\end{tabular}

Tabla 1

Factores y niveles del diseño de experimentos

\section{Resultados}

\subsection{Análisis estadístico}

La comparación de ambas políticas se realizó bajo diferentes escenarios con variación en la cantidad de pedidos y la cantidad de productos por pedido. Como era de esperarse, la figura 5 muestra que a medida que aumenta la cantidad de órdenes o de productos por orden, aumenta el tiempo requerido para su recolección en tienda. Por otra parte, se observa que, si la cantidad máxima de productos por pedido es 10, el total de horas hombre bajo la modalidad de entrega del mismo día es similar para las diferentes cantidades de pedidos. Esto sucede por los períodos ociosos que se genera por la duración del tiempo entre llegadas de las órdenes.

Adicionalmente, la figura 5 muestra que en todos los escenarios, los tiempos requeridos para el picking son inferiores bajo la promesa de entrega durante el día siguiente. Lo anterior se confirmó mediante pruebas de hipótesis de comparaciones múltiples de medias usando la corrección de Bonferroni. Como resultado se concluye, bajo todos los escenarios, que la alternativa de entrega en el día después es significativamente mejor con un valor $\mathrm{p}<0.001$.

\subsection{Análisis de costos}

Los costos incurridos bajo cada una de las políticas son medidos únicamente a través de las horas hombre de los operarios. Tomando como referencia un salario mínimo mensual vigente en Colombia más una carga prestacional del $70 \%$ y una base de 48 horas de trabajo a la semana, la hora hombre tiene un costo de 6.100 COP. Los costos obtenidos para cada política se muestran en la tabla 2. 


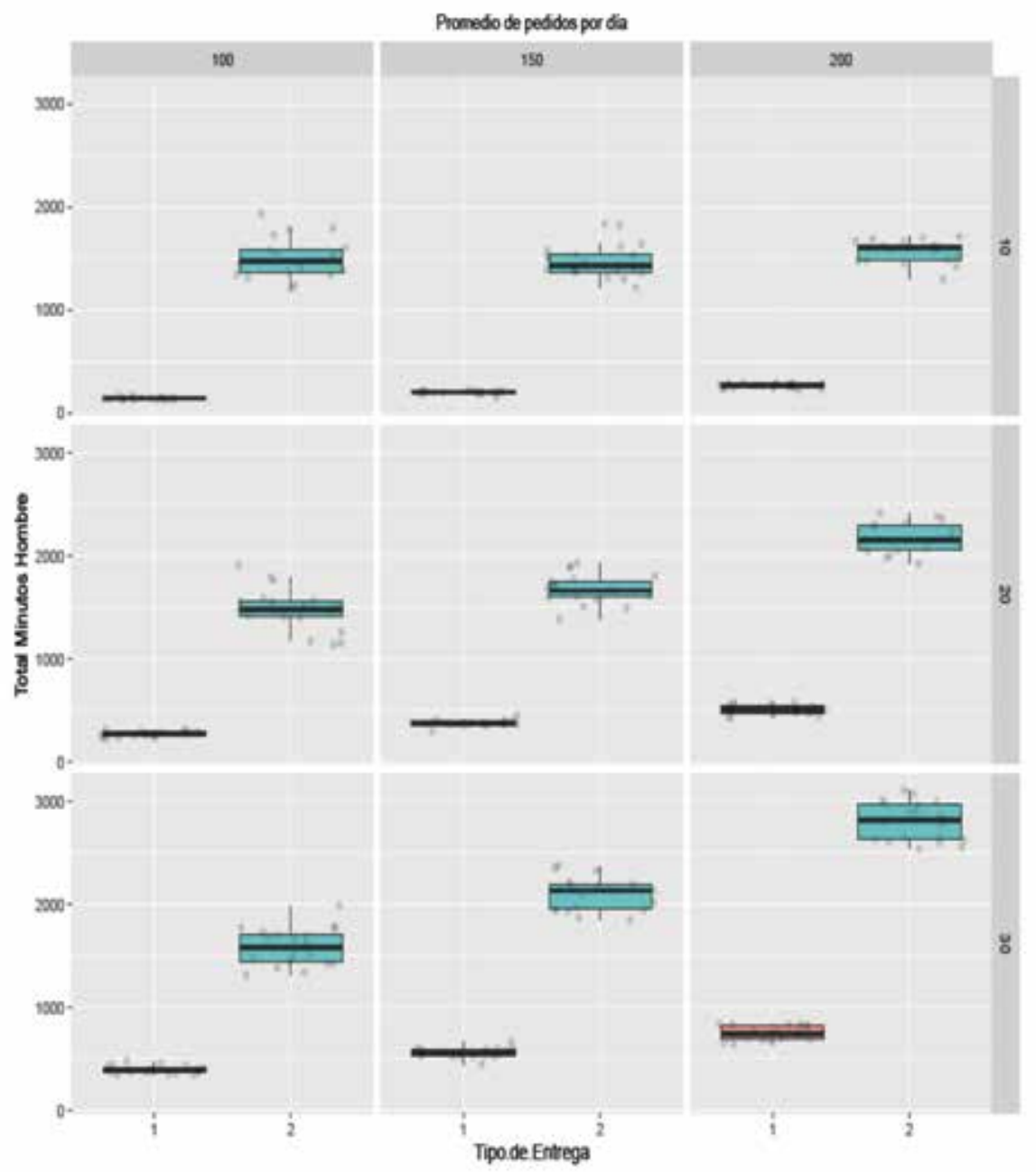

\section{Gráfico 5}

Diagrama de cajas de los resultados del experimento

Fuente: elaboración propia

Según la tabla 2, por ejemplo, bajo una tasa de 100 pedidos por día y una cantidad máxima de 10 productos por cliente, implementar la estrategia de entrega durante el día siguiente, representa en promedio, una disminución por día de 22.7 horas hombre dedicadas al picking. Bajo todos los escenarios, los costos son significativamente superiores en la política de entrega del mismo día.
A medida que la cantidad de pedidos y número de productos es mayor, el ahorro de horas hombre se incrementa al implementar la política del día siguiente. Es evidente que al tener tiempo de consolidar los pedidos de tal forma que se disminuya la distancia a recorrer en cada viaje, considerablemente, se disminuye la cantidad de horas hombre dedicadas al proceso de picking. 
COMPARACIÓN A TRAVÉS DEL PICKING / R. OTERO, S. BOLÍVAR, N. RINCóN / 591

\begin{tabular}{ccccc}
\hline $\begin{array}{c}\text { Tasa de } \\
\text { pedidos } \\
\text { por día } \\
(\lambda)\end{array}$ & $\begin{array}{c}\text { Cantidad } \\
\text { máxima de } \\
\text { productos por } \\
\text { pedido }\end{array}$ & $\begin{array}{c}\text { Diferencia promedio } \\
\text { en horas hombre } \\
\text { Alternativa (2) } \\
\text { Alternativa (1) }\end{array}$ & $\begin{array}{c}\text { Diferencia } \\
\text { promedio en COP }\end{array}$ & $\begin{array}{c}\text { Diferencia } \\
\text { porcentual } \\
(\%)\end{array}$ \\
\hline 100 & 10 & 22,7 & $\$ 138.267$ & 944 \\
100 & 20 & 20,2 & $\$ 123.118$ & 449 \\
100 & 30 & 19,8 & $\$ 120.983$ & 299 \\
150 & 10 & 21,3 & $\$ 129.930$ & 636 \\
150 & 20 & 21,7 & $\$ 132.167$ & 346 \\
150 & 30 & 25,6 & $\$ 156.262$ & 274 \\
200 & 10 & 21,8 & $\$ 132.980$ & 495 \\
200 & 20 & 27,7 & $\$ 168.767$ & 328 \\
200 & 30 & 34,2 & $\$ 208.620$ & 273 \\
\hline \multicolumn{5}{r}{} \\
\hline
\end{tabular}

Tabla 2

Diferencia en costos de las políticas de entrega por día en solo una sede

\section{Conclusiones}

En este artículo se realizó la medición del impacto de dos diferentes promesas de entrega al cliente desde el costo de las horas hombre necesarias para realizar el picking en tienda. Los resultados de los experimentos computacionales nos brindan indicios sobre los costos de ambas políticas bajo diferentes escenarios que varían la cantidad y conformación de los pedidos. En particular, bajo cualquier escenario evaluado, la promesa de entregar los productos al cliente durante el mismo día, incrementa los costos de las horas hombre en el proceso de picking en un $450 \%$ en promedio.

Esto sugiere que contar con un tiempo suficiente para consolidar todas las órdenes de los clientes, de tal forma que se logre disminuir los viajes necesarios, disminuye considerablemente la cantidad de horas hombre necesarias para la recolección de pedidos. En detalle, esta disminución se obtiene al tener que viajar un número de veces menor al punto de acopio de las órdenes y al generar rutas de viaje más inteligentes que permitan aprovechar los desplazamientos de los operarios para recolectar la mayor cantidad de productos posible.

Hasta el momento, esta investigación ha comparado tan solo dos diferentes escenarios bajo una misma política de consolidación de pedidos. Investigaciones futuras deben validar estos resultados en diferentes distribuciones de estanterías y diferentes heurísticas. Adicionalmente, es deseable incluir restricciones en el orden de recolección de productos para contemplar la categoría de productos frescos, que usualmente, se recogen al final de cada ruta para disminuir el tiempo de interrupción de la cadena de frío. Además, la experimentación con datos basados en el comportamiento real 
de compra de los clientes, usando bases de datos de los supermercados, puede generar información adicional de interés que permita apoyar la comprensión de los modelos de rentabilidad en el segmento de las entregas de productos de supermercados a domicilio.

\section{Referencias}

Armbruster, Dieter \& Gel, Esma S. (2006). Bucket Brigades Revisited: Are They Always Effective? European Journal of Operational Research, 172 (1), 213-229. http://doi. org/10.1016/j.ejor.2004.08.044

Bartholdi, John J. \& Eisenstein, Donald D. (1996). A Production Line that Balance Itself. Operations Research, 44 (1), Special Issue on New Directions in Operations Management, 21-34.

Boyer, Kenneth K. \& Hult, G. Thomas M. (2006). Customer Behavioral Intentions for Online Purchases: An Examination of Fulfillment Method and Customer Experience Level. Journal of Operations Management, 24 (2), 124-147. http://doi. org/10.1016/j.jom.2005.04.002

Bühler, Dominic; Klein, Robert \& Neugebauer, Michael (2016). Model-Based Delivery Cost Approximation in Attended Home Services. Computers \& Industrial Engineering, 98 (C), 78-90. http://doi.org/10.1016/j. cie.2016.05.014

Cámara Colombiana de Comercio Electrónico, CCCE (2016). Estudio Observatorio de compra online - Segunda oleada, Colombia 2016. Bogotá. Disponible en: https://www. ccce.org.co/sites/default/files/biblioteca/ Infograf\%C3\%ADa\%20.pdf

Chen, Yi-zhou; Shen, Shi Fei; Chen, Tao \& Yang, Rui (2014). Path Optimization Study for Vehicles Evacuation Based on Dijkstra Algorithm. Procedia Engineering, 71, 159-165. http://doi. org/10.1016/j.proeng.2014.04.023. Disponible en: https://www.researchgate. net/publication/262769062_Path_ Optimization_Study_for_Vehicles_ Evacuation_based_on_Dijkstra_Algorithm Duin, J. H. Ron van; Goffau, Wim de; Wiegmans, Bart; Tavasszy, Lori A. \& Saes, Maurice (2016). Improving Home Delivery Efficiency by Using Principles of Address Intelligence for B2C Deliveries. Transportation Research Procedia, 12, 14-25. http://doi.org/10.1016/j.trpro.2016.02.006. Disponible en: http://repository.tudelft.nl/ islandora/object/uuid\%3Aa2f956b9-57ad4f41-ad09-df318acf7234

Durand, Bruno \& González-Feliu, Jesús (2012). Urban Logistics and E-Grocery: Have Proximity Delivery Services a Positive Impact on Shopping Trips? Procedia - Social and Behavioral Sciences, 39, 510-520. http:// doi.org/10.1016/j.sbspro.2012.03.126

Ehmke, Jan Fabian \& Campbell, Ann Melissa (2014). Customer Acceptance Mechanisms for Home Deliveries in Metropolitan Areas. European Journal of Operational Research, 233 (1), 193-207. http://doi. org/10.1016/j.ejor.2013.08.028. Disponible en: https://www.researchgate.net/profile/ Ann_Campbell4/publication/270992093_ Customer_acceptance_mechanisms_for_ 
home_deliveries_in_metropolitan_areas/ links/561e607208aef097132c1b49/

Customer-acceptance-mechanisms-forhome-deliveries-in-metropolitan-areas.pdf Hall, Randolph W. (1993). Distance

Approximations for Routing Manual Pickers in a Warehouse. IIE Transactions, 25 (4), 76-87. http://doi. org/10.1080/07408179308964306

Henn, Sebastian \& Wäscher, Gerhard (2012). Tabu Search Heuristics for the Order Batching Problem in Manual Order Picking Systems. European Journal of Operational Research, 222 (3), 484-494. http://doi. org/10.1016/j.ejor.2012.05.049

Hong, Soondo; Johnson, Andrew L. \& Peters, Brett A. (2012). Batch Picking in Narrow-Aisle Order Picking Systems with Consideration for Picker Blocking. European Journal of Operational Research, 221 (3), 557-570. http://doi. org/10.1016/j.ejor.2012.03.045. Disponible en: https://www.researchgate.net/ publication/257196172_Batch_picking_in_ narrow-aisle_order_picking_systems_with_ consideration_for_picker_blocking

Hong, Soondo \& Kim, Youngjoo (2017). A

Route-Selecting Order Batching Model with the S-Shape Routes in a Parallel-Aisle Order Picking System. European Journal of Operational Research, 257 (1), 185-196. http://doi.org/10.1016/j.ejor.2016.07.017 Hsu, Chih-Ming; Chen, Kai-Ying \& Chen, Mu-Chen (2005). Batching Orders in Warehouses by Minimizing Travel Distance with Genetic Algorithms. Computers in Industry, 56 (2), 169 -
178. http://doi.org/10.1016/j. compind.2004.06.001

Hwang, Heung Suk \& Cho, Gyu Sung (2006). A Performance Evaluation Model for Order Picking Warehouse Design. Computers and Industrial Engineering, 51 (2), 335-342. http://doi.org/10.1016/j.cie.2005.10.002 Javelin Group (2011). How Many Stores Will We Really Need? UK Non-Food Retailing in 2020. Disponible en: http://www.javelingroup. com/white_paper/white_paper_ registration_how_many_stores/

Koo, Pyung-Hoi (2009). The Use of Bucket Brigades in Zone Order Picking Systems. OR Spectrum, 31 (4), 759-774. http://doi. org/10.1007/s00291-008-0131-x

Koster, René de (1994). Performance Approximation of Pick-To-Belt Order Picking Systems. European Journal of Operational Research, 72 (3), 558573. http://doi.org/10.1016/03772217(94)90423-5. Disponible en: https:// repub.eur.nl/pub/11836/PerformanceAppro ximation_1994pdf.pdf

Koster, René de; Le-Duc, Tho \& Roodbergen, Kees Jan (2007). Design and Control of Warehouse Order Picking: A Literature Review. European Journal of Operational Research, 182 (2), 481-501. http://doi. org/10.1016/j.ejor.2006.07.009. Disponible en: http://roodbergen.com/publications/ EJOR2007.pdf

Le-Duc, Tho \& Koster, René de (2007). Travel Time Estimation and Order Batching in a 2-Block Warehouse. European Journal of Operational Research, 176 (1), 374-388. http://doi.org/10.1016/j.ejor.2005.03.052 
Liao, Shu-hsien; Chen, Yin-ju \& Lin, Yi-tsun (2011). Mining Customer Knowledge to Implement Online Shopping and Home Delivery for Hypermarkets. Expert Systems with Applications, 38 (4), 3982-3991. http://doi.org/10.1016/j.eswa.2010.09.059. Disponible en: ftp://140.131.110.38/leecc/ public/SPSS\&AppofStatistics/2011Papers/ Reference/Mining\%20customer\%20 knowledge\%20to\%20implement\%20 online $\% 20$ shopping\%20and $\% 20$ home $\% 20$ delivery\%20for\%20hypermarkets.pdf Martello, Silvano \& Toth, Paolo (1987). Algorithms for Knapsack Problems. NorthHolland Mathematics Studies, 132 (C), 213-257. http://doi.org/10.1016/S03040208(08)73237-7

Pan, Jason Chao-Hsien; Shih, Po-Hsun \& Wu, Ming-Hung (2015). Order Batching in a Pick-and-Pass Warehousing System with Group Genetic Algorithm. Omega, 57, 238-248. http://doi.org/10.1016/j. omega.2015.05.004

Park, Minyoung \& Regan, Amelia (2004). Issues in Emerging Home Delivery Operations. University of California Transportation Center, 2 (2), 1-13. http://doi. org/10.1068/a201285

Rincón-García, Nicolás (2016). Freight Transport, Routing Software and TimeDependent Vehicle Routing Models. Doctoral Thesis. University of Southampton, Faculty of Engineering and the Environment, Southampton, England. Disponible en: https://eprints. soton.ac.uk/397141/1/FINAL\%2520E-
THESIS\%2520FOR\%2520EPRINTS\%252025739344.pdf

Saskia, Seidel; Mareï, Nora \& Blanquart, Corinne (2016). Innovations in e-Grocery and Logistics Solutions for Cities. Transportation Research Procedia, 12, 825-835. http://doi.org/10.1016/j. trpro.2016.02.035

Visser, Johan; Nemoto, Toshinori \& Browne, Michael (2014). Home Delivery and the Impacts on Urban Freight Transport: A Review. Procedia - Social and Behavioral Sciences, 125, 15-27. http://doi.org/10.1016/j. sbspro.2014.01.1452

Yu, Mengfei \& Koster, René de (2010). Enhancing Performance in Order Picking Processes by Dynamic Storage Systems. International Journal of Production Research, 48 (16), 4785-4806. http://doi. org/10.1080/00207540903055693

- Fecha de recepción: 12 de abril de 2016

- Fecha de aceptación: 11 de junio de 2016

- Disponible en línea: 14 de diciembre de 2016

\section{Para citar este artículo}

Otero-Caicedo, Ricardo; Bolívar, Stevenson \& Rincón-García, Nicolás (2016). Comparación a través del picking en tienda de dos alternativas de entrega en un entorno de servicio a domicilio en supermercados. Área temática: Logística en ciudad. Cuadernos de Contabilidad, 17 (44), 575594. 